e-mail: ferdinand.schmidt-kaler@uibk.ac.at, FAX: $+435125072952$

Key words Quantum computing, quantum bits, entanglement, single ions 


\title{
How to realize a universal quantum gate with trapped ions
}

\author{
Ferdinand Schmidt-Kaler *, Hartmut Häffner, Stephan Gulde, Mark Riebe, Gavin P.T. Lancaster, \\ Thomas Deuschle, Christoph Becher, Wolfgang Hänsel, Jürgen Eschner, Christian F. Roos, and \\ Rainer Blatt
}

Institut für Experimentalphysik, Universität Innsbruck, Technikerstraße 25, A-6020 Innsbruck, Austria

Received: date / Revised version: date

\begin{abstract}
We report the realization of an elementary quantum processor based on a linear crystal of trapped ions. Each ion serves as a quantum bit (qubit) to store the quantum information in long lived electronic states. We present the realization of single-qubit and of universal two-qubit logic gates. The two-qubit operation relies on the coupling of the ions through their collective quantized motion. A detailed description of the setup and the methods is included.
\end{abstract}

\section{Introduction}

Quantum computers (QC) are known to perform certain computational tasks more efficiently than their classical counterparts. The theoretical concept of QC is highly developed. Most well-known among the quantum algorithms [1] is the efficient algorithm for the factorization of large numbers 2 which threatens the security of the commonly used RSA-encryption scheme. Furthermore, efficient quantum algorithms exist for searching entries in an unsorted data base [3, for simulating quantum spin systems [4, and for quantum games. As in a classical computer, errors will necessarily occur. Although the nature of errors is different in quantum mechanical and in classical computers, algorithms have been developed which can correct qubit errors [5] 6. World-wide efforts aim at a scalable realization of a QC [7]. Already in 1995, J. I. Cirac and P. Zoller proposed to implement a scalable QC on a string of trapped ions, where each ion's electronic state represents a qubit 8 . Quantum gates between any subset of ions would be induced by laser-ion interactions, including the coupling of the ions to their collective quantized motion [9]. Today, a number of different proposals for quantum gates in an ion based QC are known.

While the construction of a large scale QC might still be in remote future, we may already today perform

\footnotetext{
* corresponding author
}

experiments with a small number of qubits, bringing into reality what used to be Gedanken experiments and thus enlightening the foundations of quantum mechanics. This will serve to further extend our knowledge of the puzzling quantum theory and its borderline to classical physics, given by decoherence and the measurement process [10].

The ion-trap system itself is fully understood theoretically, and equally well its interaction with a laser field. Any kind of quantum logic gate operation may thus be predicted. Actual experiments are performed with few ions that are confined in a Paul trap, such that time scales for decoherence and for the dephasing of qubits due to fluctuations of external parameters are long as compared to the coherent qubit operation times. The detection of the ions' internal states relies on electron shelving, leading to a detection efficiency near unity. In this kind of fully defined, text-book like setting, elementary quantum processors may be realized. Quantum logic gate operations and entangled states may be studied.

The most challenging experimental step towards achieving the Cirac\&Zoller scheme (CZ) of a QC is to implement the controlled-NOT (CNOT) gate operation between two individual ions. The CNOT quantum logical gate corresponds to the XOR gate operation of classical logic which flips the state of a target bit conditioned on the state of a control bit. Taking the basis states $|a, b\rangle=$ $\{|0,0\rangle,|0,1\rangle,|1,0\rangle,|1,1\rangle\}$ of two qubits, the CNOT operation reads $|a, b\rangle \rightarrow|a, a \oplus b\rangle$, where $\oplus$ represents an addition modulo 2 . Only if the control qubit (first entry) is in $|1\rangle$, the quantum state of the control qubit changes. Here, we present the realization of a CNOT quantum gate [11] according to the original $\mathrm{CZ}$ proposal [8].

In our experiment, two ${ }^{40} \mathrm{Ca}^{+}$ions are held in a linear Paul trap and are individually addressed with focussed laser beams. Superpositions of long-lived electronic states represent a qubit. By initializing the control and target qubit in all four basis states and performing the CNOT operation, we determine the desired truth table. To prove the quantum nature of the gate, we use a 
superposition state for the control qubit and generate an entangled output state.

The paper gives a detailed description of the experimental apparatus and the required procedures in sections 2 and 3 In sect. 4 we discuss the realization of the universal two-ion CNOT gate, followed by a discussion of its current limitations and possible future improvements.

\section{Experimental setup}

\subsection{Levels and transitions in the ${ }^{40} \mathrm{Ca}^{+}$ion}

The Calcium ion $\left({ }^{40} \mathrm{Ca}^{+}\right)$has a single valence electron and no hyperfine structure, see fig. Th for the relevant levels and transitions. We have chosen ${ }^{40} \mathrm{Ca}^{+}$for several reasons: (a) The transition wavelengths for Dopplercooling and optical pumping are well suited for solidstate and diode laser sources. (b) Long-lived metastable states $(\tau \sim 1 \mathrm{~s})$ allow for the implementation of qubits. (c) The narrow-line quadrupole transition can also be used to implement sideband cooling to the vibrational ground state.

We cool the ion on the $\mathrm{S}_{1 / 2}$ to $\mathrm{P}_{1 / 2}$ transition near $397 \mathrm{~nm}$ close to the Doppler limit. The UV-radiation is produced as the second harmonic of a Ti:Sapphire laser at $794 \mathrm{~nm}^{1}$. Grating stabilized diode lasers at $866 \mathrm{~nm}$ and $854 \mathrm{~nm}$ prevent pumping into the $\mathrm{D}_{3 / 2}$ and $\mathrm{D}_{5 / 2}$ states. Each of the above lasers is frequency-locked to its individual optical reference cavity using the Pound-DreverHall method [14. With cavity linewidths of 2-5 MHz, we reach a laser frequency stability of better than $300 \mathrm{kHz}$. Frequency tuning of the lasers is achieved by scanning the length of the corresponding reference cavities using piezo-electric actuators.

The electronic level $\mathrm{S}_{1 / 2}(m=-1 / 2) \equiv|S\rangle$ is identified with logic $|0\rangle$ and $\mathrm{D}_{5 / 2}(m=-1 / 2) \equiv|D\rangle$ with $\operatorname{logic}|1\rangle$, respectively. To perform quantum logic operations, we excite the corresponding transition with a Ti:Sapphire laser near $729 \mathrm{~nm}$. The complete laser system for the qubit manipulation is described in sect. 2.4 and 2.5.

We detect the quantum state of the qubit by applying the laser beams at $397 \mathrm{~nm}$ and $866 \mathrm{~nm}$ and monitoring the fluorescence of the ion at $397 \mathrm{~nm}$ on a photomultiplier and on a CCD camera (electron shelving technique [15]). The internal state of the ion is discriminated with an efficiency close to $100 \%$, details of the detection are found in sect. 3.5

It is of advantage that pure ${ }^{40} \mathrm{Ca}^{+}$ion crystals can be loaded into the trap using a relatively simple photoionization scheme 16 that relies on a two step laser excitation: A weak beam of neutral Ca is emitted by a resistantly heated oven [17. Calcium atoms are excited on

1 The practicability of a grating stabilized UV-diode 1213 for single ion cooling and detection has been proven.
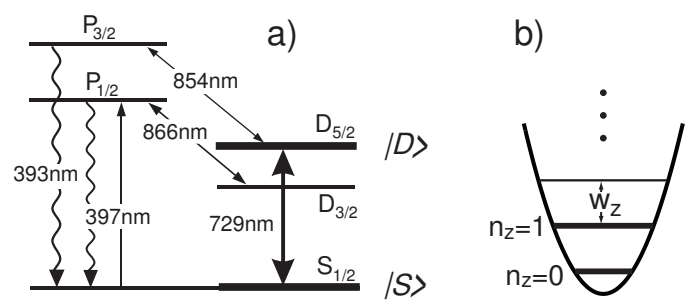

Fig. 1 a ${ }^{40} \mathrm{Ca}^{+}$level scheme. A qubit is encoded in the $S_{1 / 2},(m=-1 / 2)$ ground and $D_{5 / 2},(m=-1 / 2)$ metastable state of a single trapped ion. b) The lowest two number states $n$ of an axial vibrational motion in the trap are used as quantum bus.

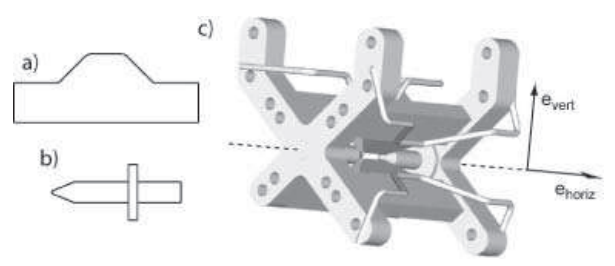

Fig. 2 Construction of the linear trap 19] out of four blades (a) and two tips (b). The 3D-view (c) shows the arrangement of the RF-blades which generate the radial trapping potential. The closest distance between the blades is $1.6 \mathrm{~mm}$. The tips are separated by $5.0 \mathrm{~mm}$. All electrodes are mounted onto a Macor ceramics spacer. The typical machining precision of all parts is 5 to $10 \mu \mathrm{m}$. The RF-blades are fabricated by electro-erosion from stainless steel, the tips are made of molybdenum.

the $4 s^{1} S_{0} \rightarrow 4 p^{1} P_{1}$ transition near $423 \mathrm{~nm}$ by a grating stabilized diode laser 18 17. Ionization is reached with radiation at $\lambda \leq 390 \mathrm{~nm}$ using a UV-diode laser or even a simple UV-light emitting diode.

\subsection{Linear Paul trap}

For the experiments, ${ }^{40} \mathrm{Ca}^{+}$ions are stored in the harmonic potential of a linear Paul trap. The trap is made of four blades for radial confinement and two tips for axial confinement, see fig. 2. Under typical operating conditions we observe axial and radial motional frequencies $\left(\omega_{\mathrm{ax}}, \omega_{\mathrm{rad}}\right) / 2 \pi=(1.2,5.0) \mathrm{MHz}$, respectively. The trap combines good optical access with relatively high trapping frequencies, even though the trap dimensions are comparatively large. Electrically insulating parts have no direct line of sight to the ions. We attribute the low heating rate $(<1$ phonon/50ms) 20 to the combination of a large distance between ions and trap electrodes $\left(\mathrm{r}_{0} \approx 0.8 \mathrm{~mm}\right)$ and the clean loading scheme by photo-ionization. Both tips, typically at $+1 \mathrm{kV}$, are positioned in the symmetry axis with high precision. Small asymmetries are compensated by applying voltages of below $200 \mathrm{~V}$ to electrodes which are placed at a radial distance of $30 \mathrm{~mm}$ from the trap symmetry axis (fig. 2.2). The radio frequency $(\mathrm{RF}) \Omega /(2 \pi) \simeq 23.5 \mathrm{MHz}$ is applied 
to two diagonally opposing blades (the other two being at $0 \mathrm{~V}$ ). This creates an oscillating electrical quadrupole field which results in a radial trapping potential. The RF is generated by a synthesizer ${ }^{2}$ and amplified ${ }^{3}$ to $15 \mathrm{~W}$. A helical $\lambda$ /4-resonator (loaded Q-value $\sim 200$ ) serves to match the capacitive load of the trap structure with the $50 \Omega$ output of the amplifier and to enhance the drive voltage to a few $\mathrm{kV}_{p p}$. We typically operate the trap close to the stability parameter $\mathrm{q} \leq 0.6[21$. In order to avoid RF pick-up on the DC-voltage leads we use separate feed-throughs and filter the DC voltages.

The trap is mounted in a UHV housing, pumped by a Titanium sublimation and an ion getter pump ${ }^{4}$. The residual gas pressure is below $2 \times 10^{-11}$ mbar.

\subsection{Optical setup}

The output of a Ti:Sapphire laser ${ }^{5}$ near $794 \mathrm{~nm}$ is frequencydoubled $^{6}$ to obtain up to $50 \mathrm{~mW}$ light at $397 \mathrm{~nm}$. We stabilize the UV power to $1 \%(\mathrm{rms})$ using an AOM in front of the doubling cavity. During a gate operation on the qubit transition, any residual UV-light has to be suppressed to a maximum. As the UV-light needs to be switched faster than mechanical shutters would allow, we pass it through an $\mathrm{AOM}^{7}$, couple into a single-mode polarization-maintaining fibre $^{8}$ and transport it to the trap. After the fiber output, the light is sent through a second AOM. Switching the RF-drive of both AOM's yields an extinction of about $2 \times 10^{-6}$. Additionally, due to the fibre, the UV-beam is spatially filtered such that its focus on the ion crystal $\left(w_{0} \sim 50 \mu \mathrm{m}, \leq 100 \mu \mathrm{W}\right)$ does not cause excessive stray-light on the trap electrodes. The UV-beam leaving the second AOM is split into two beams which are superimposed with light at $866 \mathrm{~nm}$ and $854 \mathrm{~nm}$. These beams enter the vacuum system via UVAR coated windows, and intersect at the ion trap under angles of $\left\{-22_{\text {hor. }}^{\circ}, 0_{\text {vert. }}^{\circ}\right\}$ with respect to the axial trap direction, and $\left\{22_{\text {hor. }}^{\circ}, 45_{\text {vert. }}^{\circ}\right\}$, respectively. The combination of both light fields is used for Doppler cooling, ion detection, and the compensation of micro-motion.

Another part of the UV-light transmitted through the fiber is controlled by a third AOM, enters along the axis of the magnetic field $\left\{22_{\text {hor. }}^{\circ}, 0_{\text {vert }}^{\circ}\right\}$, and is applied for optical pumping.

The switching of the light field at $854 \mathrm{~nm}$ is controlled by an additional AOM in double-pass configuration to assure on/off-dynamics of about $2 \times 10^{-4}$. The laser field at $866 \mathrm{~nm}$ does not couple to the qubit levels and is kept on continuously.

\footnotetext{
${ }^{2}$ Marconi Inc., Signal gen. 2019A

3 Minicircuits Inc., LZY-1

4 Varian Inc., Starcell 20

5 Coherent Inc., 899-21

6 Spectra Inc., Wavetrain

7 Brimrose Inc., QZF-80-20

8 Schäfter Kirchhof Inc.
}

The fluorescence of the ions at $397 \mathrm{~nm}$ is collected through a viewport using a large collimating lens ${ }^{9}$ at a working distance of $65 \mathrm{~mm}$ and focused onto an intensified CCD camera ${ }^{10}$. This corresponds to a solid angle of 0.01 of $4 \pi$. A magnification of $\times 20$ is chosen. In opposite direction, a similar lens with magnification of $\times 7$ is used for single photon counting with a photomultiplier ${ }^{11}$ (PMT). We estimate an overall detection efficiency of $0.1 \%$ and $0.2 \%$ for the CCD and PMT, respectively. We typically obtain a PMT count rate of $\sim 30 \mathrm{kHz}$ from a single ion, while the stray light level is below $2 \mathrm{kHz}$. The direction of the detection with respect to the trap symmetry axis is $\left\{-68_{\text {hor. }}^{\circ}, 0_{\text {vert. }}^{\circ}\right\}$ and $\left\{112_{\text {hor. }}^{\circ}, 0_{\text {vert. }}^{\circ}\right\}$ for CCD and PMT, respectively.

\subsection{Laser setup for the qubit transition}

Qubit operations are performed with laser light near $729 \mathrm{~nm}$, generated from a second Ti:Sapphire laser ${ }^{12}$. To obtain a high fidelity of gate operations this laser source has to be stabilized in frequency and intensity to a high degree. For frequency stability, we rely on a stable reference cavity. Its length stability is guaranteed by a spacer from ultra-low thermal expansion material (ULE) on which the cavity mirrors (super-mirrors with a few ppm loss and transmission, measured finesse of $2.4 \times 10^{5}$ ) are optically contacted. For additional stability, the cavity is suspended on wires in a temperature stabilized UHV chamber. We derive a Pound-DreverHall error signal 14 and stabilize the laser frequency with a servo bandwidth of $\leq 2.5 \mathrm{MHz}$ obtaining a laser linewidth $\leq 100 \mathrm{~Hz}[22$. The laser intensity is stabilized using an AOM to about $1 \%$ (rms).

The qubit operations require laser pulses with well defined phase, frequency, intensity and duration. We modulate the output of the Ti:Sapphire laser $(\sim 350 \mathrm{~mW})$ with an $\mathrm{AOM}^{13}$ (see fig. 3) in double-pass configuration. The radio frequencies and phases that are applied to the AOM transfer directly to the light field ${ }^{14}$.

For maximum flexibility of the complex temporal pattern, we use the scheme depicted in fig. 3. Sideband ground state cooling is performed with sources F4 and F5 at frequencies resonant to the red sidebands $\omega_{0}$ and $\sqrt{3} \omega_{0}$. The specific quantum gate sequence is composed of pulses on the carrier and blue sideband of the bus mode, driven by the sources F1 and F2, while F3 is used for the AC-Stark compensation (see sect. 3.3). The computer digital output card ${ }^{15}$, temporal resolution $1 \mu \mathrm{s}, 32$

\footnotetext{
${ }^{9}$ Nikon, MNH-23150-ED-Plan-1.5x

10 Princton Instum., Inc. I-Penta-MAX

11 Electron-Tubes Inc., P25

12 Coherent Inc., 899-21

13 Brimrose Inc.,TEF-270-100

14 Due to the double-pass configuration, the modulation of laser frequency and phase is twice the applied RF modulation.

15 Jäger Inc., ADwin
} 


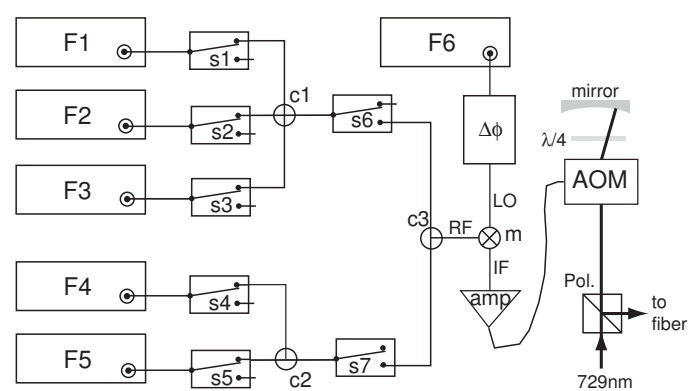

Fig. 3 RF setup for control of $729 \mathrm{~nm}$ laser: The output of RF-synthesizers $\mathrm{F} 1$ to $\mathrm{F} 5^{16}$ is controlled by switches $(s 1$ to $s 7)^{17}$, added up with combiners $(c 1 \text { to } c 3)^{18}$ and mixed ${ }^{19}$ with the output of the synthesizer $F 6$, which serves as local oscillator (LO). The phase of the LO is controlled via a digital phase shifter(DPS) ${ }^{20}$. The output (IF) is amplified and fed to an acousto-optical modulator, operated in doublepass configuration. The frequencies $\mathrm{F} 1$ to $\mathrm{F} 6$ are computer controlled via GPIB.

channels, serves to switch the frequency sources and the digital phase shifter. F6 compensates for the drift of the laser reference cavity. The linear drift component of $\leq$ $10 \mathrm{~Hz} / \mathrm{s}$ is determined by comparison to the atomic resonance and anticipatively corrected for. As we saturate the LO input of the frequency mixer $m$, a small RFtransmission modulation of the phase shifter for different $\Delta \phi$ does not convert into a RF-intensity modulation at IF. In addition, this RF-setup allows for the generation of multi-chromatic light fields, as necessary e.g. for the compensation of the AC-Stark effect.

\subsection{Single ion addressing optics}

For addressing individual ions, light at $729 \mathrm{~nm}$ is spatially filtered and transported by an optical fibre. A twolens telescope expands the $729 \mathrm{~nm}$ beam while an electrooptic deflector(EOD) ${ }^{21}$ in front of the lenses controls the beam direction. We direct this expanded laser beam $\left(\mathrm{w}_{0} \sim 1 \mathrm{~cm}\right)$ counterpropagating to the emerging fluorescence towards the CCD, using a dichroic beam splitter and focus it onto individual ions by using the same lens ${ }^{22}$ as for imaging the fluorescence light. The focused beam of up to $80 \mathrm{~mW}$ hits the ion crystal under an angle of $\left\{68_{\text {hor. }}^{\circ}, 0_{\text {vert. }}^{\circ}\right\}$. The corresponding single ion Lamb-Dicke factors are $\eta_{\text {axial }}=0.033$ and $\eta_{\text {radial }}=0.040$, respectively. By varying the voltage applied to the EOD we

\footnotetext{
16 Marconi Inc., Signal gen. 2023

17 Minicircuits Inc., ZYSW-2-50DR

18 Minicircuits Inc., ZSC-2-1

19 Minicircuits Inc., ZP-2

20 Lorch Inc., DP-1-8-370-5-77

21 LaserComponents Inc., ED2-730

22 Nikon, MNH-23150-ED-Plan-1.5x
}

steer the focus at the ion position by more than $10 \mu \mathrm{m}$, large compared to the two-ion distance of $4.90 \mu \mathrm{m}^{23}$ for $\omega_{\mathrm{ax}} /(2 \pi)=1.2 \mathrm{MHz}$. Additionally, the high-voltage controller for the deflector can be preset to values which are selected through digital input lines. The digital signals are computer-generated by the same digital output board that controls the RF pulses. Between different addressing positions, we typically leave a settling time of $15 \mu \mathrm{s}$. The determination of the spatial resolution is discussed in sect. 3.2

In order to provide a quantization axis and to split the Zeeman components of the $\mathrm{S}_{1 / 2}$ to $\mathrm{D}_{5 / 2}$ transition, we compensate the ambient magnetic field and generate a constant magnetic field of $2.4 \mathrm{G}$ under an angle of $\left\{22_{\text {hor. }}^{\circ}, 0_{\text {vert. }}^{\circ}\right\}$ which is thus perpendicular to the $\vec{k}$ vector of the addressing light field. The geometry and polarization of the light field at $729 \mathrm{~nm}$ allows the excitation of $\Delta m=0, \pm 1$ and \pm 2 transitions 23 .

\section{Preparative procedures and measurements}

This section addresses the methods that are used to prepare and manipulate the ions for a typical experimental sequence. In a first step, the ions are initialized in a well defined state using sympathetic sideband cooling and optical pumping (sect. 3.1). Then, the ions are individually manipulated on the qubit transition. During manipulation on the sideband frequencies, the level-shifts due to the AC-Stark effect need to be counteracted by additional laser frequencies (sect. 3.3). Finally, the individual states of the ions are detected by means of a CCD camera and a PMT (sect. 3.5.5).

\subsection{Ground state cooling}

Each experimental cycle starts with the preparation of the ions in a well defined initial state. The motional state of the two ion crystal can be described by 6 different vibrational modes 24]. The axial and two radial centerof-mass modes at $\omega_{\mathrm{ax}}$ and $\omega_{\text {rad }}^{(x, y)}$ coincide with the singleion trap frequencies. The two radial rocking modes and one axial breathing mode have frequencies of $\omega_{\mathrm{R}}^{(x, y)}=$ $\sqrt{\omega_{\text {rad }}^{(x, y)} 2-\omega_{\mathrm{ax}}^{2}}$ and $\omega_{\mathrm{b}}=\sqrt{3} \omega_{\mathrm{ax}}$, respectively. In our experiment, we have chosen the breathing mode as the 'bus-mode' for the quantum gate and we therefore need to prepare it in the ground state $\left|n_{\mathrm{b}}=0\right\rangle$. For the radial spectator modes, Doppler cooling is sufficient as $\eta_{\mathrm{rad}}=$ $0.04 \ll 1^{24}$. However, the axial spectator mode 25] at $\omega_{\text {ax }}$ is sideband cooled in addition to the bus-mode.

The cooling cycle starts with a $2 \mathrm{~ms}$ period of Doppler cooling on the $\mathrm{S}_{1 / 2}$ to $\mathrm{P}_{1 / 2}$ transition at $397 \mathrm{~nm}$ dur-

\footnotetext{
23 Projection of the two-ion distance of $5.29 \mu \mathrm{m}$ under $22^{\circ}$

24 The final temperature is close to the Doppler cooling limit if the UV light intensity is below saturation
} 
ing which the repumping laser on the $\mathrm{D}_{3 / 2}$ to $\mathrm{P}_{1 / 2}$ line $(866 \mathrm{~nm})$ is switched on.

After a short period of optical pumping into the $\mathrm{S}_{1 / 2}\left(m_{J}=\right.$ $-1 / 2$ ) state (typically $30 \mu \mathrm{s}$ ), the bus-mode and the axial center of mass mode are sequentially sideband cooled using the quadrupole transition at $729 \mathrm{~nm}$ 20. We switch the $729 \mathrm{~nm}$-laser to one of the two ions and subsequently perform a cooling cycle for $2 \mathrm{~ms}$ and $6 \mathrm{~ms}$ on the red sideband of the center-of-mass mode and of the bus-mode, respectively. The cooling rate is enhanced to several $\mathrm{kHz}$ by a quench laser on the $\mathrm{D}_{5 / 2}$ to $\mathrm{P}_{3 / 2}$ transition at $854 \mathrm{~nm}$. During these periods, the $\sigma^{-}$-beam is repeatedly pulsed on every $2 \mathrm{~ms}$ to recollect atoms that have been pumped to the $\mathrm{S}_{1 / 2}\left(m_{J}=+1 / 2\right)$ state.

With this procedure, we achieve a ground state population of the bus-mode of about 99\% [26 20] and a coefficient of $\eta^{2} \bar{n} \ll 1$ for all spectator modes. At the end of the cooling cycle, a last optical pumping pulse initializes the ion chain in the electronic ground state $\mathrm{S}_{1 / 2}\left(m_{J}=-1 / 2\right)$.

\subsection{Addressing single ions}

As explained in section 2.5. the focus position of the manipulation laser at $729 \mathrm{~nm}$ is controlled by the EOD.

The quality of the addressing can be evaluated from Rabi oscillations between the $\mathrm{S}_{1 / 2}\left(m_{J}=-1 / 2\right)$ and the $\mathrm{D}_{5 / 2}\left(m_{J}=-1 / 2\right)$ state, that are driven on one out of two ions. Residual laser light that reaches the second ion leads to a slow Rabi oscillation of the second ion. From such measurement, we infer the addressing error, i.e. the amount of unwanted qubit rotation on the second ion which is present during a particular one-qubit manipulation on the first ion, and vice versa. Fig. 4 shows two typical excitation patterns for such Rabi flops.

It is important to note that this addressing error does not fundamentally limit the accuracy of one-qubit rotations. For the current experiments, we have included this effect in the error budget [11. It is, however, possible to counteract the unwanted rotation on the second ion by an additional laser pulse that is addressed to the second ion. The remaining error on the first ion would then be of second order, and even this contribution could be eliminated by a clever choice of pulses. To make such counteraction possible, one has to determine the phase difference between the laser light addressed directly to ion 1 and the residual light that generates the unwanted rotation on ion 1 while the beam is addressed to ion 2 .

We have measured this phase difference with only one ion in the trap. For this, we adjust the beam such that, without deflection, the ion is centrally addressed. Figure 5 shows the dependence of the Rabi frequency on the beam deflection. The corresponding laser intensity is approximately given by a Gaussian with a waist of $2.5 \mu \mathrm{m}[19$. We now perform a spin-echo experiment with the laser frequency tuned to the carrier transition.
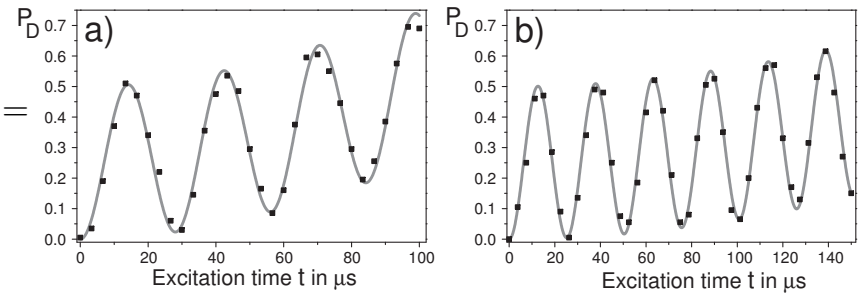

Fig. 4 Rabi oscillations on the carrier, performed on a twoion crystal after ground state sideband cooling of both the axial COM and the breathing mode. The plots show the average excitation into the $|D\rangle$ state $\left(\left(P_{D, 1}+P_{D, 2}\right) / 2\right)$ which is measured with the PMT. For the data shown in (a), the laser is addressed onto the first of the two ions, for (b) onto the second one. For the given adjustment of the optics, the addressing error is different for the two ions: (a) $\Omega_{1}=2 \pi \cdot 35.5(1) \mathrm{kHz}, \Omega_{2}=2 \pi \cdot 2.46(7) \mathrm{kHz}$. We find an addressing error $\Omega_{1} / \Omega_{2}=6.9(1) \%$, and a ratio of light intensities of 1:210. (b) $\Omega_{1}=2 \pi \cdot 39.7(2) \mathrm{kHz}, \Omega_{2}=2 \pi \cdot 1.16(5) \mathrm{kHz}$, which corresponds to a ratio of Rabi frequencies of $2.9(1) \%$, and 1:1200 for the light intensities.

The two framing pulses are performed with a deflected beam and with the controlled phase set to 0 and $\pi$, respectively. Because of the beam deflection, the ion feels the laser phase $\Delta \Phi$ and $\Delta \Phi+\pi$. The center pulse is directly addressed to the ion, with the controlled phase set to $\phi$. If we define $R^{x}(\theta, \Phi)$ 27] to be the qubit rotation by an angle $\theta$ about the horizontal axis characterized by the polar angle $\Phi$, where $x$ denotes the beam deflection, then the action on the atom can be described by:

$$
R_{\text {echo }}^{x}=R^{x}\left(\frac{\pi}{2}, \Delta \Phi\right) R^{0}(\pi, \Phi) R^{x}\left(\frac{\pi}{2}, \Delta \Phi+\pi\right)
$$

If the phase difference between the deflected and the addressed beam $\Delta \Phi$ is equal to zero, then we expect no spin flip for a phase $\Phi= \pm \pi / 2$. Moreover, scanning the phase $\Phi$ yields an excitation from the $|S\rangle$ to the $|D\rangle$ state of $P_{D}=\cos ^{2}(\Phi-\Delta \Phi)$ which can be fitted to infer the phase shift $\Delta \Phi$. The dependence of this phase shift on the beam deflection is shown in fig. 5 b.

We attribute the linear part of the phase shift to the elongation of the optical path within the EOD. Such behavior is expected if the laser beam is not ideally aligned with the EOD axis. For beam deflections larger than $\pm 2 \mu \mathrm{m}, \Delta \Phi$ depends no longer in a linear way on the deflection. We suppose that this is due to light which does not travel through the optical system along the ideal path and therefore has a phase different from the Gaussian part of the beam. Such a hypothesis is supported by the small pedestal below the Gaussian profile in fig. 5

As an important result we note that the phase difference between the deflected and the addressed beam is well defined and stable over long periods of time, even for large deflections. This offers the possibility to reduce the effect of the addressing error in future experiments. 


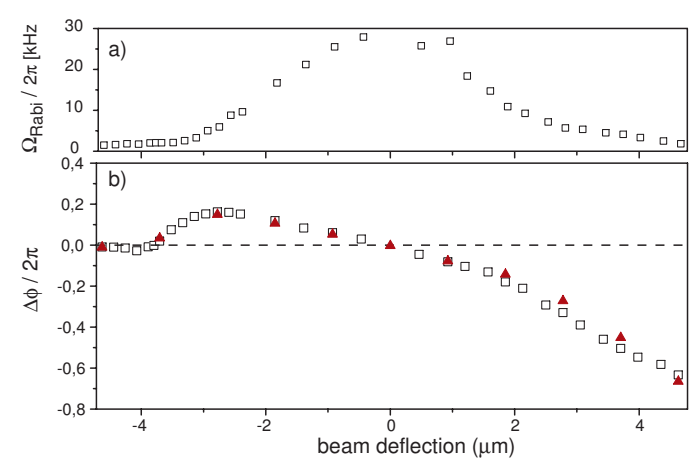

Fig. 5 (a) Rabi frequency of the ion as a function of the deflector voltage $U_{\text {defl }}$.

(b)Phase difference $\Delta \Phi$ between the addressed $\left(U_{\text {defl }}=0\right)$ and the deflected beam, as perceived at the ion's position. The different symbols represent independent measurements. The data stem from a measuring period of more than 4 hours.

\subsection{AC Stark compensation}

As the ions which represent the qubits are not ideal twolevel systems, the manipulation of the qubit states can be perturbed by non-resonant coupling to other levels. In particular, for manipulations on the vibrational sideband, the coupling to the carrier 28 is so strong that it induces important light shifts (AC Stark shifts) on the qubit levels. As this would perturb their phases, the light shift needs to be compensated by an additional laser frequency of appropriate power and detuning [29]. The compensating light field is generated by a frequency F3, also applied to the double-pass AOM in the $729 \mathrm{~nm}$ beam (see section 2.4). Using the same laser beam as a source, this setup ensures that laser power fluctuations or changes in the beam alignment are not converted into phase fluctuations.

\subsection{Phase gate and composite laser pulses}

The central quantum-logic operation in the Cirac-Zoller CNOT-gate is a one-ion phase gate where the sign of the electronic qubit is switched conditional on the vibrational state. In the computational subspace $(|D, 0\rangle$, $|D, 1\rangle,|S, 0\rangle,|S, 1\rangle)$, this gate is described by a diagonal matrix with the entries $(1,-1,-1,-1)^{25}$.

Excitation on the blue motional sideband leads to a pairwise coupling between levels $|S, n\rangle \leftrightarrow|D, n+1\rangle$ except for the level $|D, 0\rangle$. For the phase gate we perform an effective $2 \pi$-pulse on the two two-level systems $(|S, 0\rangle \leftrightarrow|D, 1\rangle)$ and $(|S, 1\rangle \leftrightarrow|D, 2\rangle)$ which changes the sign of all computational basis states except for $|D, 0\rangle)$. Since the Rabi frequency depends on $n$, we need to use a composite-pulse sequence 30] instead of a single blue

\footnotetext{
25 This transformation is the standard phase gate up to an
} overall phase factor of -1 .
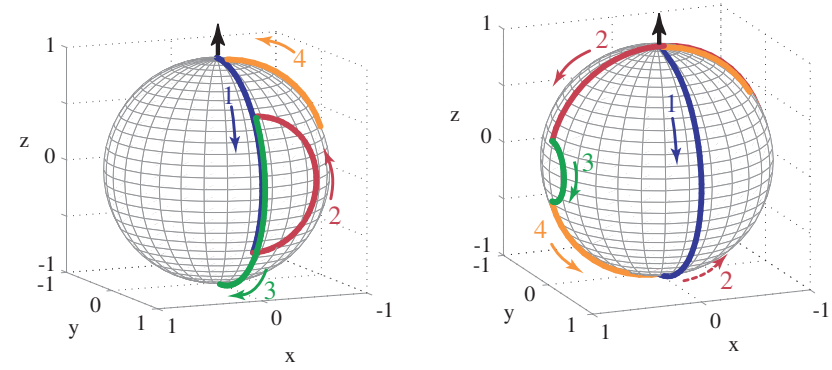

Fig. 6 Bloch sphere trajectories for the composite phase gate, $R_{\text {phase }}$ Left: Bloch sphere for the quasi-two-levelsystem $|S, 0\rangle \leftrightarrow|D, 1\rangle$. The initial state is $|S, 0\rangle$, indicated by the black arrow. Pulse $R_{1}$ of the sequence rotates the state vector about the $x$-axis by $\pi / \sqrt{2} . R_{2}$ accomplishes a $\pi$-rotation about the $y$-axis. It therefore transforms the state to its mirror image about the $x$ - $y$-plane. Consequently, $R_{3}$, which is identical to $R_{1}$, rotates the state vector all the way down to the bottom of the sphere. $R_{4}$, just like $R_{2}$, represents a $\pi$-rotation about the $y$-axis. The final state identical to the initial one, except the acquired phase factor -1 .

Right: The same laser pulse sequence acting in the $|S, 1\rangle \leftrightarrow$ $|D, 2\rangle$ subspace. Again, the final state identical to the initial one, except the acquired phase factor -1 .

sideband pulse. The sequence is composed of four sideband pulses $R_{4} R_{3} R_{2} R_{1}$ and can be described by

$$
\begin{aligned}
R_{\text {phase }}= & R^{+}(\pi \sqrt{n+1}, 0) R^{+}\left(\pi \sqrt{\frac{n+1}{2}}, \pi / 2\right) \\
& \cdot R^{+}(\pi \sqrt{n+1}, 0) R^{+}\left(\pi \sqrt{\frac{n+1}{2}}, \pi / 2\right)
\end{aligned}
$$

where $n$ denotes the lower vibrational quantum number of the two coupled states and $R^{+}[27$, similar to $R$ in equation 1 denotes a rotation induced by coupling to the upper motional sideband. Figure 6 illustrates the evolution of the Bloch vectors during the phase gate and provides a step-by-step picture of the process ${ }^{26}$.

It may be helpful to interpret this evolution in terms of spin-echos. For the system $(|S, 0\rangle \leftrightarrow|D, 1\rangle)$, the first three pulses constitute a spin-echo experiment where the $\pi$-pulse in the middle assures that the overall evolution is the one of a $\pi$-pulse, despite the rotation angle of $\pi / \sqrt{2}$. This evolution is followed by an additional $\pi$ pulse which completes the $2 \pi$-rotation. For the second twolevel system $(|S, 1\rangle \leftrightarrow|D, 2\rangle)$, the sequence starts with a $\pi$-pulse that is followed by the spin-echo-type $\pi$-rotation.

The phase gate is transformed into a CNOT operation if it is sandwiched in between two $\pi / 2$ carrier pulses, $R_{\mathrm{CNOT}}=R(\pi / 2,0) R_{\text {phase }} R(\pi / 2, \pi)$.

\subsection{Qubit readout}

For detection of the internal quantum states, we excite the $\mathrm{S}_{1 / 2}$ to $\mathrm{P}_{1 / 2}$ dipole transition near $397 \mathrm{~nm}$ and mon-

26 The Bloch-sphere picture doesn't give complete information on the phases picked up during the evolution. Those have to be computed using a matrix representation. 